Virginia Commonwealth University VCU Scholars Compass

\title{
The effect of substituted benzene dicarboxylic acid linkers on the optical band gap energy and magnetic coupling in manganese trimer metal organic frameworks
}

K.S. Asha

Indian Institute of Science Education and Research Thiruvananthapuram

Arthur C. Reber

Virginia Commonwealth University, acreber@vcu.edu

N. Ahmed

Indian Institute of Science Education and Research Thiruvananthapuram

See next page for additional authors

Follow this and additional works at: http://scholarscompass.vcu.edu/phys_pubs

Part of the Physics Commons

This journal is (C) The Royal Society of Chemistry 2017

\section{Downloaded from}

http://scholarscompass.vcu.edu/phys_pubs/212

This Article is brought to you for free and open access by the Dept. of Physics at VCU Scholars Compass. It has been accepted for inclusion in Physics Publications by an authorized administrator of VCU Scholars Compass. For more information, please contact libcompass@vcu.edu. 
Authors

K. S. Asha, Arthur C. Reber, N. Ahmed, R. Nath, Shiv N. Khanna, and Sukhendu Mandal 


\section{Journal of Materials Chemistry C} Materials for optical, magnetic and electronic devices rsc.li/materials-c

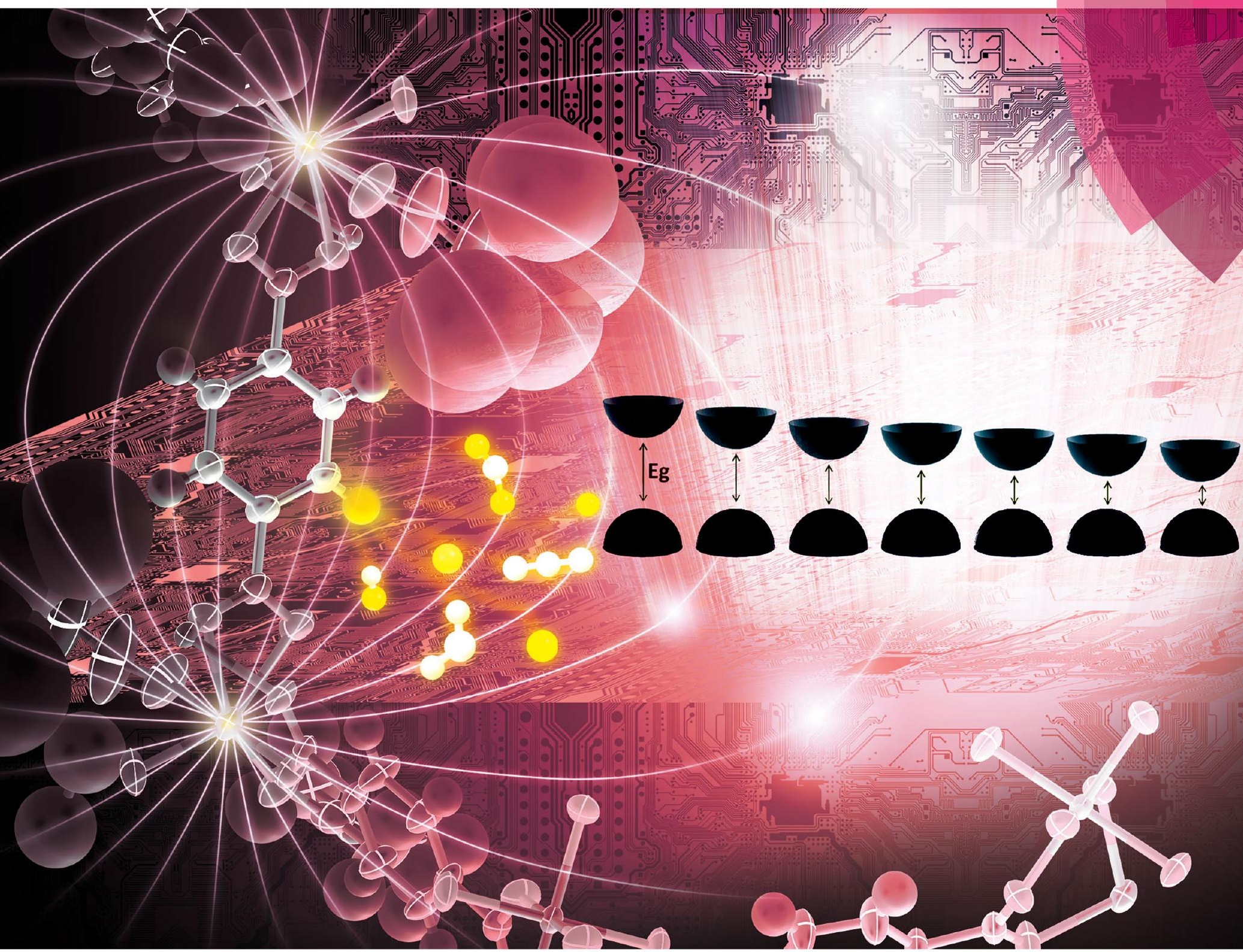

ISSN 2050-7526 


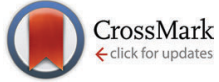

Cite this: J. Mater. Chem. C, 2017, 5,539

Received 19th August 2016,

Accepted 9th November 2016

DOI: $10.1039 / c 6 t c 03577 a$

www.rsc.org/MaterialsC

\title{
The effect of substituted benzene dicarboxylic acid linkers on the optical band gap energy and magnetic coupling in manganese trimer metal organic frameworks $\dagger$
}

\author{
K. S. Asha, ${ }^{a}$ Arthur C. Reber, ${ }^{b}$ N. Ahmed, ${ }^{c}$ R. Nath, ${ }^{* c}$ Shiv N. Khanna*b and \\ Sukhendu Mandak ${ }^{*}$
}

\begin{abstract}
We have systematically studied a series of eight metal-organic frameworks (MOFs) in which the secondary building unit is a manganese trimer cluster, and the linkers are differently substituted benzene dicarboxylic acids (BDC). The optical band gap energy of the compounds vary from $2.62 \mathrm{eV}$ to $3.57 \mathrm{eV}$, and theoretical studies find that different functional groups result in new states in the conduction band, which lie in the gap and lower the optical band gap energy. The optical absorption between the filled $\mathrm{Mn} 3 \mathrm{~d}$ states and the ligands is weak due to minimal overlap of the states, and the measured optical band gap energy is due to transitions on the BDC linker. The Mn atoms in the MOFs have local moments of $5 \mu_{\mathrm{B}}$, and selected MOFs are found to be antiferromagnetic, with weak coupling between the cluster units, and paramagnetic above $10 \mathrm{~K}$.
\end{abstract}

\section{Introduction}

Metal-organic frameworks (MOFs) represent an important class of cluster solids that are synthesized by linking metal atoms or clusters with organic linkers. ${ }^{1-5}$ The choice of organic ligand allows for the formation of frameworks with different length scales and different interactions between the metal centers. ${ }^{6-9}$ MOFs in which the metal centers have a magnetic moment are of particular interest as the distance between the magnetic motifs and sometimes the linkers may offer a unique opportunity to tune the magnetic properties. ${ }^{10-24}$ Manganese based compounds are of particular interest as a $\mathrm{Mn}$ atom has an electronic configuration of $3 \mathrm{~d}^{5} 4 \mathrm{~s}^{2}$ with a filled majority $3 \mathrm{~d}^{5}$ shell and large exchange splitting leading to a robust atomic moment. ${ }^{11-15,25,26}$ The stable moment of $\mathrm{Mn}$ led to the first molecular magnet composed of $\mathrm{Mn}_{12} \mathrm{O}_{12}$ clusters linked by acetate and water. ${ }^{27,28}$

\footnotetext{
${ }^{a}$ School of Chemistry, Indian Institute of Science Education and Research Thiruvananthapuram, Thiruvananthapuram, Kerala-695016, India. E-mail: sukhendu@iisertvm.ac.in

${ }^{b}$ Department of Physics, Virginia Commonwealth University, Richmond, VA, USA. E-mail: snkhanna@vcu.edu

${ }^{c}$ School of Physics, Indian Institute of Science Education and Research Thiruvananthapuram, Thiruvananthapuram, Kerala-695016, India. E-mail: rnath@iisertvm.ac.in

$\dagger$ Electronic supplementary information (ESI) available: Synthesis, characterization including SCXRD, PXRD, IR, and TGA, crystallographic parameter table, optical absorption spectra of the $\mathrm{Mn}_{3}$-benzoic acid complex and the energy landscape for the magnetization direction. CCDC 1474408-1474414 (1-7). For ESI and crystallographic data in CIF or other electronic format see DOI: 10.1039/c6tc03577a
}

Each $\mathrm{Mn}_{12} \mathrm{O}_{12}$ cluster has an inner shell of four $\mathrm{Mn}$ atoms coupled antiferromagnetically to an outer shell of eight Mn atoms forming a cluster with a moment of $20 \mu_{\mathrm{B}}{ }^{29,30}$ This cluster solid was the first demonstration of space quantization at a macroscopic scale as each $\mathrm{Mn}_{12} \mathrm{O}_{12}$ cluster had a magnetic anisotropic energy of around $51 \mathrm{~K}^{28,29}$

The ability to control the optical band gap energy of a material is valuable for photocatalytic and optoelectronic applications. ${ }^{7,8,31-36}$ Understanding the features that control the band gap energy of a material requires systematic synthesis of a set of materials with variable linkers and/or metal motifs. Previous studies have shown that by varying the linker or the cluster motif, the optical band gap energy of MOFs and cluster solids may be controlled. ${ }^{7,34-38}$ Generally, one does not expect magnetic materials to have a significant band gap since the majority or minority manifolds are not fully occupied. In the present case, $\mathrm{Mn}^{2+}$ has a $3 \mathrm{~d}^{5}$ configuration in which the majority spin shell is filled, while the minority $3 \mathrm{~d}$ shell is empty, and therefore the system could be expected to show a significant optical band gap. This raises the question of whether the optical band gap energy of Mn based materials can be controlled. The purpose of this work is to systematically study a series of Mn based metal-organic frameworks that are magnetic and have a controllable optical band gap energy as the first step towards developing multifunctional magnetic materials. Through synergistic studies of the experiments and first principles theory, we report the synthesis and characterization of eight MOFs composed of Mn trimers and benzene-dicarboxylic acid derivatives. 
By singly substituting the BDC linker with $\mathrm{F}, \mathrm{Cl}, \mathrm{Br}, \mathrm{OH}, \mathrm{NH}_{2}, \mathrm{~N}_{3}$, and $\mathrm{NO}_{2}$, the effects of the linker on the magnetic and optical properties were studied. Experimental studies indicate that the resulting solids exhibit an interesting magnetic behavior, where the susceptibility exhibits a broad feature at low temperatures for several compounds, while in other cases the susceptibility increases continuously up to $2.1 \mathrm{~K}$. These are signatures of antiferromagnetism and paramagnetism, respectively. A Curie-Weiss fit to the data reveals the effective moment and the nature of the magnetic interactions. Theoretical studies find that the materials have an antiferromagnetic ground state in which the ferrimagnetic clusters are antiferromagnetically coupled to each other with a low energy transition towards a ferrimagnetic state. The theoretical studies also show that the $\mathrm{Mn}_{3}$-based motifs are marked by strong exchange coupling and weak magnetic anisotropy, which result in super-paramagnetic relaxations above a few Kelvins. We also find that the linkers form the conduction band edge of the given series of MOFs, and that changing the functional group of the linkers, and the position of the conduction band edge, may allow tuning of the optical band gap energy.

\section{Results}

\section{Structural description and optical spectra}

We have synthesized eight new MOFs which are structurally similar. In all of them, the metal center is a manganese ion and the ligands are benzene dicarboxylic acid (BDC) or derivatives of BDC. Compounds 1-4 crystallize in a triclinic structure with the space group $P \overline{1}$, compounds 6 and 7 crystallize in a monoclinic structure with the space group $P 2_{1} / n$ and compound 5 crystallizes in a orthorhombic structure with the Pbca space group (see the ESI $\dagger$ for the crystallographic parameters). The unit cell of compounds 1-4 consists of two asymmetric units and those of compounds 6 and 7 have four units, whereas compound 5 consists of eight asymmetric units. The secondary building units (SBUs) are trimers $\left(\left(\mathrm{Mn}_{3}(\mathrm{OCO})_{6} \mathrm{O}_{4}\right)\right)$, which are linked to each other via BDC or substituted BDC forming a 2-D network.

The $\mathrm{Mn}^{2+}$ ions in a trimer unit are connected via $\mathrm{Mn}-\mathrm{O}-\mathrm{Mn}$ and $\mathrm{Mn}-\mathrm{O}-\mathrm{C}-\mathrm{O}-\mathrm{Mn}$ bonds as shown in Fig. 1a, and the end metal ions of the trimer unit are passivated by the oxygen of the two DMF molecules. The asymmetric unit of all eight structures consists of three metal ions, three ligand molecules and four coordinated solvent molecules as shown in Fig. $1 \mathrm{~b}$.

The $\mathrm{Mn}^{2+}$ ions are six coordinated with a distorted octahedral geometry. The central $\mathrm{Mn}^{2+}$ ion is coordinated to six carboxylate a)

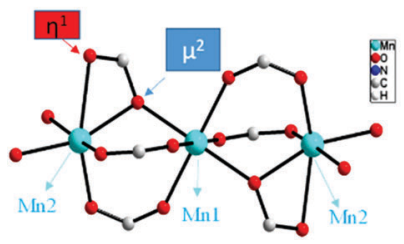

b)

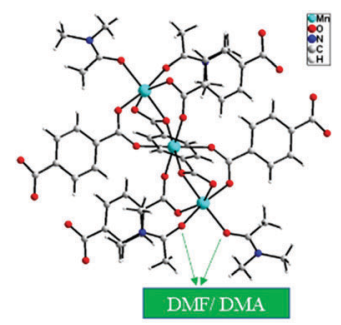

Fig. 1 (a) The SBU and (b) the asymmetric unit of compound 1. oxygen atoms whereas the two terminal $\mathrm{Mn}^{2+}$ ions are coordinated to four carboxylate oxygen atoms and two oxygens from the DMF solvent molecules (Fig. 1b). The metal clusters are linked with ligands to form the layered architectures (Fig. 2). The 2D net is then connected by non-covalent interactions between the coordinated solvent molecules forming a 3D network. The purity of all the as-synthesized compounds was validated by comparing the experimental PXRD data with the simulated data (Fig. S1, $\mathrm{ESI} \dagger$ ). The XRD pattern of compound 8 was also matched with that of compound 7, which indicates the similar structural architecture of compounds 7 and 8, respectively, as shown in Fig. S1 (ESI $\dagger$ ). The conversion of the $-\mathrm{NH}_{2}$ to $-\mathrm{N}_{3}$ based linker was proved by the IR spectra. Before $-\mathrm{N}_{3}$ conversion there was a doublet peak at 3200 and $3350 \mathrm{~cm}^{-1}$, which correspond to $-\mathrm{NH}_{2}$ stretching $\left(\gamma_{\text {sym }}\right.$ and $\left.\gamma_{\text {asym }}\right)$, and after the reaction these peaks disappear. There is also a new band formed at $2100 \mathrm{~cm}^{-1}$ due to $\mathrm{N}_{3}$ stretching (Fig. S2, ESI $\dagger$ ). The thermogravimetric analysis of all the compounds was carried out under a $\mathrm{N}_{2}$ atmosphere (Fig. S3, ESI $\dagger$ ). The optical band gap measurements of all the compounds (1-8) were determined from the diffuse reflectance of the solid state samples. The experimental $E_{\mathrm{bg}}$ values were determined from the Tauc plot and are listed in Table 1 (Fig. 3). Since the band structure of the MOFs is rather flat with little dispersion, we have calculated the band gap energies using the formula for the indirect band gap energy. The band gap energies are varied between $2.62 \mathrm{eV}$ and $3.57 \mathrm{eV}$ as the ligand functionality is changed. The main difference in structure assemblies is the ligand functionality, and how this ligand functionality creates the difference in the band gap energy is explained in detail by conducting theoretical investigations.

\section{Magnetic measurements}

Magnetic susceptibility $\chi(T)$ as a function of temperature was measured at an applied field of $H=1 \mathrm{~T}$. The magnetic isotherm $(M$ vs. $H$ ) was measured at $T=2.1 \mathrm{~K}$ by varying $H$ from 0 to $9 \mathrm{~T}$. For both the measurements, a vibrating sample magnetometer (VSM) attached to the PPMS was used. The $\chi(T)$ values of compounds 1-8 are shown in Fig. 4a, c, e, g, 5a, c, e, and g, respectively. At high temperatures, $\chi(T)$ increases with decreasing temperatures following a Curie-Weiss behavior as expected in the paramagnetic regime. Compounds 1, 2, and 6 show a broad maximum at $T^{\max } \simeq 3.16 \mathrm{~K}, 7.4 \mathrm{~K}$, and $8.5 \mathrm{~K}$, respectively, indicative of short-range antiferromagnetic ordering which is a typical feature of low-dimensional magnetic compounds. The $\mathrm{d} \chi / \mathrm{d} T$ derivative in compound 2 shows an anomaly at $T_{\mathrm{N}} \simeq 4.5 \mathrm{~K}$, suggesting the occurrence of magnetic long-range ordering (LRO), while all the other compounds do not show any signature of magnetic LRO at low temperatures. In order to extract the magnetic parameters, we performed a Curie-Weiss (CW) fit to the $1 / \chi$ vs. $T$ data at high temperatures using the following expression: ${ }^{39}$

$$
\chi(T)=\chi_{\mathrm{o}}+\frac{c}{T+\theta_{\mathrm{CW}}}
$$

where $\chi_{0}$ is temperature-independent and includes contributions from core diamagnetic susceptibility and Van-Vleck paramagnetic susceptibility. The second term is the Curie-Weiss law with $C$ being the Curie constant and $\theta_{\mathrm{CW}}$ being the characteristic 

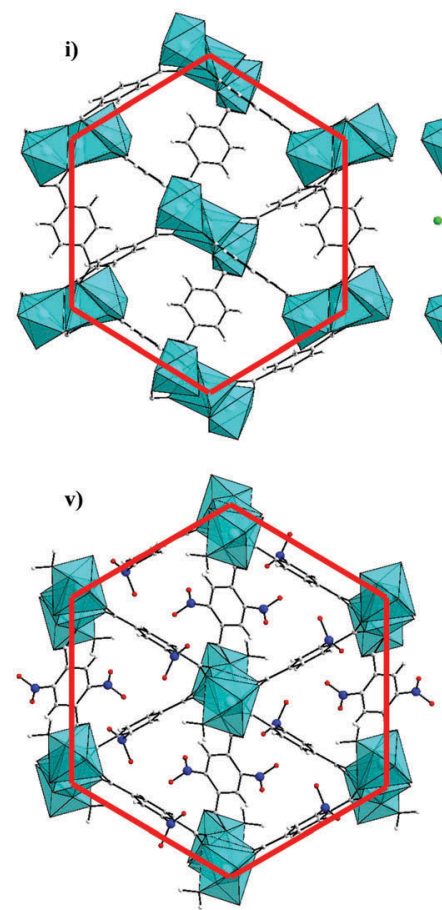
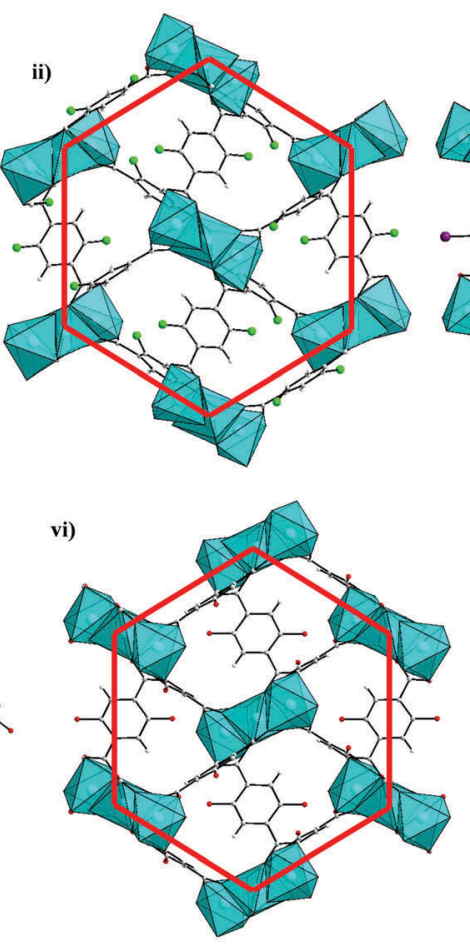
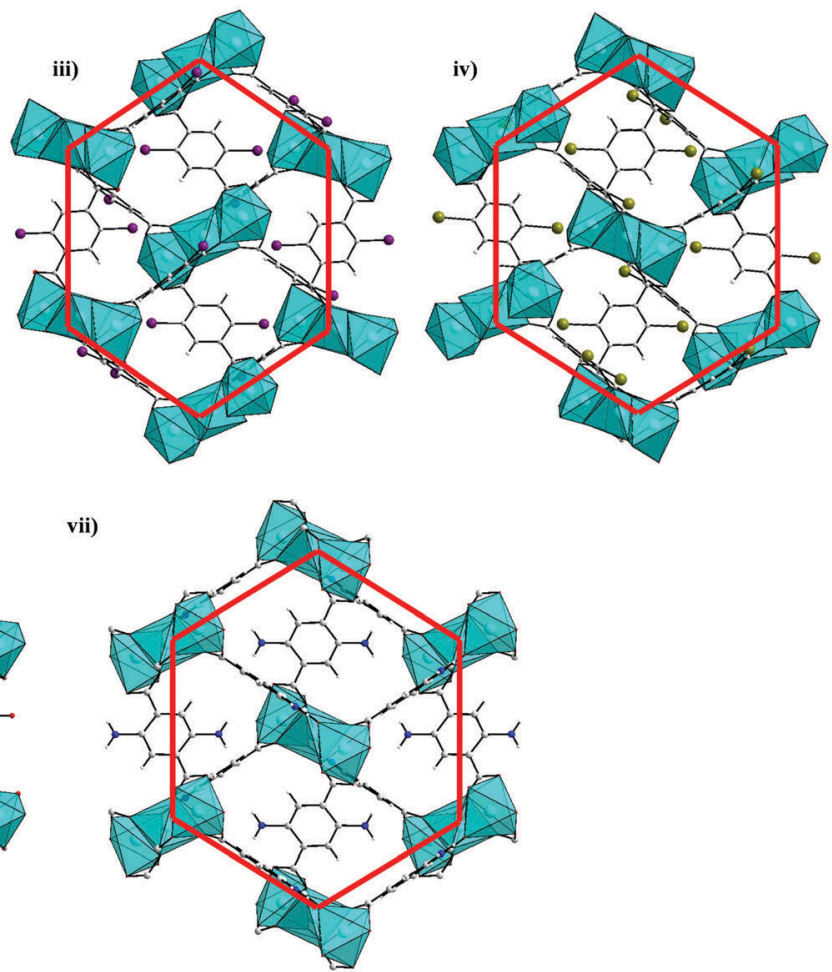

Fig. 2 The (2,6)-inter connected 3-D network of compounds 1 (i), 2 (ii), 3 (iii), 4 (iv), 5 (v), 6 (vi), and 7 (vii).

Table 1 The experimental band gap energy, and theoretical band gap energies of the solid, the optical band gap energy, and the HOMO-LUMO gap of the isolated substituted benzene-1,4-dicarboxylic acid

\begin{tabular}{llllll}
\hline $\mathrm{C}$ & $\mathrm{R}$ & $\begin{array}{l}\text { Band gap } \\
\text { exp. (eV) }\end{array}$ & $\begin{array}{l}\text { Band gap } \\
\text { alpha }(\mathrm{eV})\end{array}$ & $\begin{array}{l}\text { Optical gap } \\
\text { (beta eV) }\end{array}$ & $\begin{array}{l}\text { Molec. gap } \\
(\mathrm{eV})\end{array}$ \\
\hline $\mathbf{1}$ & $\mathrm{H}$ & 3.57 & 1.02 & 3.11 & 3.55 \\
$\mathbf{2}$ & $\mathrm{F}$ & 3.52 & 0.91 & 3.14 & 3.47 \\
$\mathbf{3}$ & $\mathrm{Cl}$ & 3.48 & 0.98 & 3.06 & 3.37 \\
$\mathbf{4}$ & $\mathrm{Br}$ & 2.87 & 0.95 & 3.01 & 3.27 \\
$\mathbf{5}$ & $\mathrm{NO}_{2}$ & 3.17 & 0.31 & 2.50 & 3.13 \\
$\mathbf{6}$ & $\mathrm{OH}$ & 2.93 & 1.16 & 2.63 & 3.07 \\
7 & $\mathrm{NH}_{2}$ & 2.73 & 1.01 & 2.19 & 2.54 \\
$\mathbf{8}$ & $\mathrm{N}_{3}$ & 2.62 & 0.85 & 2.70 & 2.94
\end{tabular}

Curie-Weiss temperature. Fig. 4b, d, f, h, 5b, d, f, and h show the Curie-Weiss fit to the $1 / \chi$ data at high temperatures, which was extrapolated down to low temperatures for compounds $\mathbf{1}, \mathbf{2}$, $\mathbf{3}, \mathbf{4}, \mathbf{5}, \mathbf{6 ,} \mathbf{7}$, and 8, respectively. Parameters obtained from the CW fit [eqn (1)] are tabulated in Table 2. The calculated effective moments are in close agreement with the expected spin-only value of $\mu_{\text {eff }}=g \sqrt{S(S+1)} \mu_{\mathrm{B}} \simeq 5.92 \mu_{\mathrm{B}}$ for $\mathrm{Mn}^{2+}(S=5 / 2) .{ }^{40}$ The slight differences between the expected and calculated values in certain compounds are likely due to a small difference in the Landé $g$-values, as mentioned in Table 2. According to the mean field theory, $\theta_{\mathrm{CW}}$ is the sum of all the exchange interactions. Thus the positive value of $\theta_{\mathrm{CW}}$ for all the compounds suggests a dominant antiferromagnetic super-exchange interaction between the $\mathrm{Mn}^{2+}$ ions. ${ }^{39}$ Except for compound 1, all the other compounds have comparatively large $\theta_{\mathrm{CW}}$ values, indicating a strong exchange interaction between the $\mathrm{Mn}^{2+}$ ions.

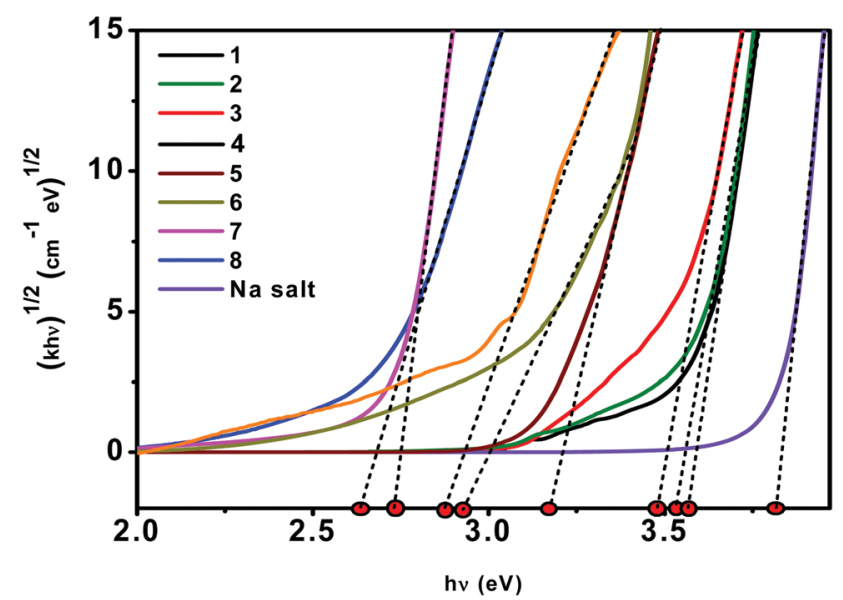

Fig. 3 The Tauc plot of compounds $\mathbf{1 - 8}$ and sodium salt of the BDC ${ }^{2-}$ ligand.

The insets of Fig. $4 \mathrm{~b}, \mathrm{~d}, \mathrm{f}, \mathrm{h}, 5 \mathrm{~b}, \mathrm{~d}, \mathrm{f}$, and $\mathrm{h}$ show the magnetization isotherm ( $M$ vs. $H$ ) at $T=2.1 \mathrm{~K}$ for compounds $\mathbf{1 - 8}$, respectively. For compound $1, M$ is found to saturate at a $H_{\mathrm{S}} \simeq$ of $4.5 \mathrm{~T}$ with a saturation magnetization $M_{\text {sat }}$ of $\simeq 5.02 \mu_{\mathrm{B}}$, which is close to the expected saturation magnetization value of $M_{\mathrm{S}}=g S \mu_{\mathrm{B}}=5 \mu_{\mathrm{B}}$ for $S=5 / 2$. For all other compounds, saturation couldn't be achieved at $H=9$ T. Compounds 3, 4, 5, 7, and 8 exhibit a plateau at a magnetization value of $\simeq 1.75 \mu_{\mathrm{B}}$, which is much lower than the expected saturation value of $M_{\mathrm{S}}=5 \mu_{\mathrm{B}}$. This is close to the expected magnetic moment for a ferrimagnetic trimer when two $\mathrm{Mn}$ atoms have up and one $\mathrm{Mn}$ atom has down 

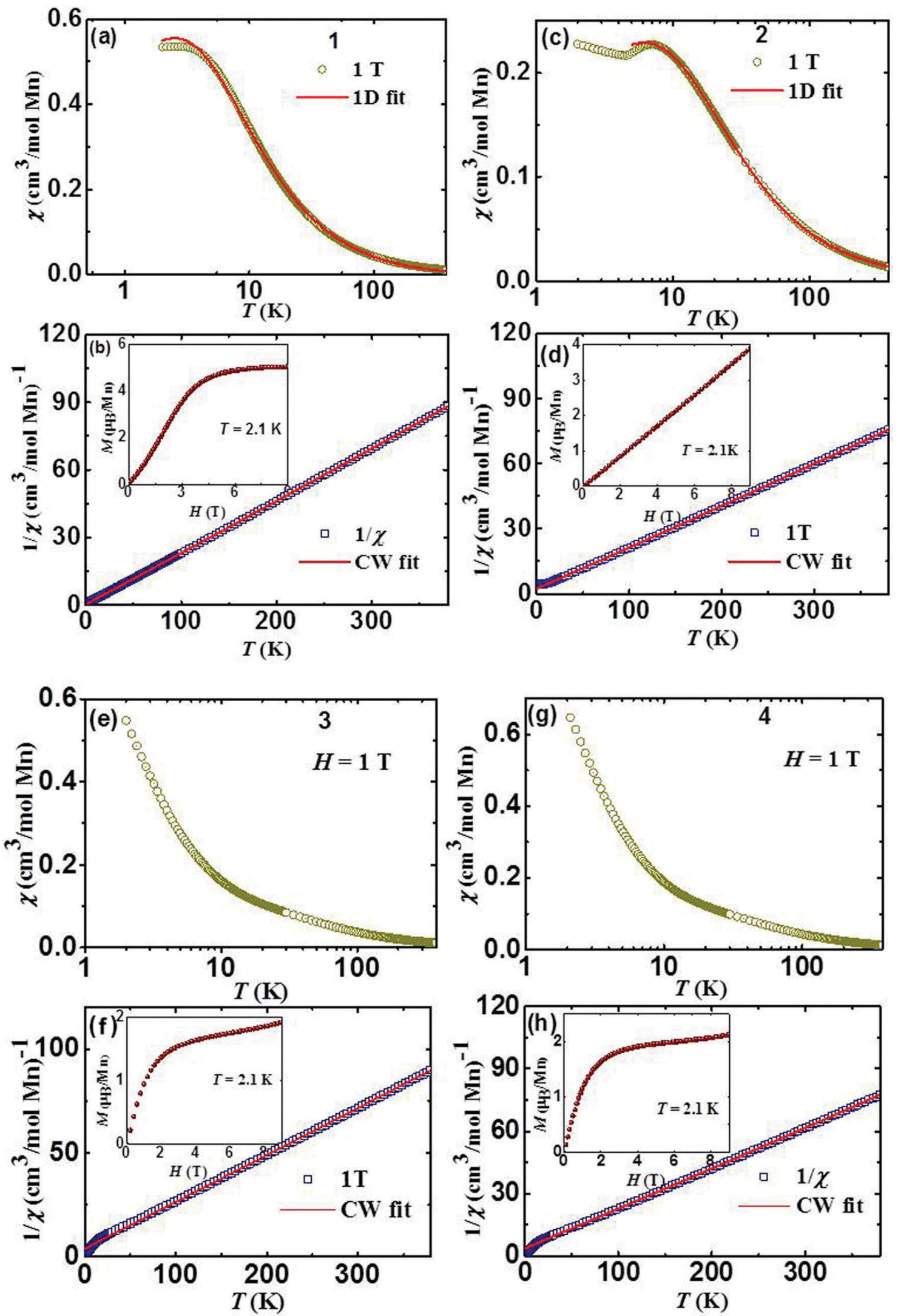

Fig. 4 Upper panels: Magnetic susceptibility $(\chi)$ vs. temperature $(T)$ of compounds $1-4$ measured at an applied field $H=1 \mathrm{~T}$. The solid line represents the 1D fit as described in the text. Lower panels: $1 / \chi v$ s. $T$ at an applied field $H=1 \mathrm{~T}$. The solid line is the CW fit at high temperatures using eqn (1). Inset: The magnetization isotherm $M(H)$ measured at $T=2.1 \mathrm{~K}$. 

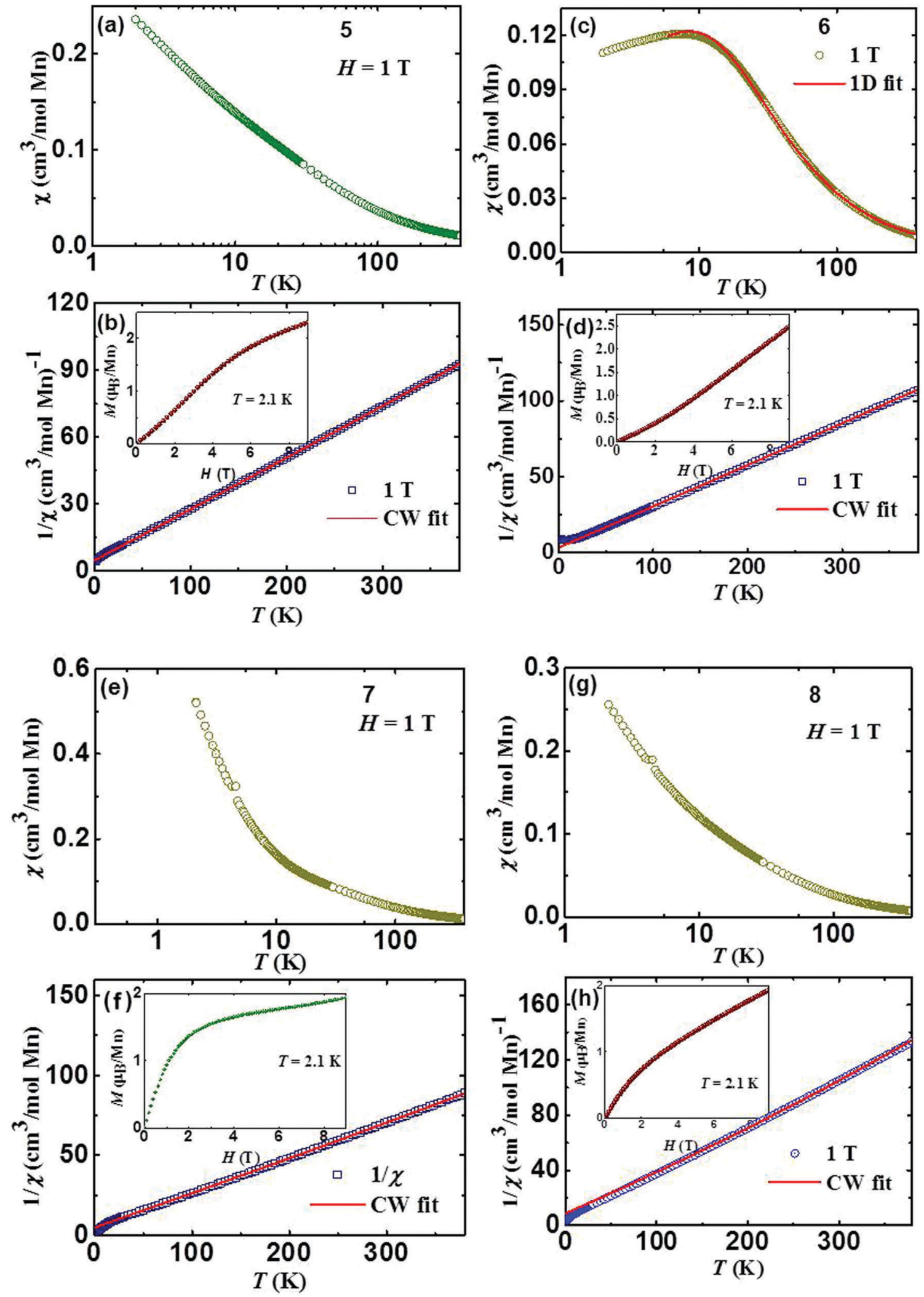

Fig. 5 Upper panels: $\chi$ vs. $T$ of compounds 5-8 at $H=1 \mathrm{~T}$. Lower panels: $1 / \chi$ vs. $T$ at $H=1 \mathrm{~T}$. The solid line is the $\mathrm{CW}$ fit at high temperatures using eqn (1). Inset: The magnetization isotherm $M(H)$ measured at $T=2.1 \mathrm{~K}$.

spin configurations, as this results in a magnetic moment of completely flat plateau state is possibly due to dominant $5 \mu_{\mathrm{B}}$ per three Mn atoms, or $5 / 3 \mu_{\mathrm{B}}$ per Mn atom. The lack of a Van-Vleck paramagnetism. ${ }^{41}$ For compounds 2 and $6, M$ almost 
Table 2 Magnetic parameters obtained from the CW fit [eqn (1)] to the $1 / \chi(T)$ data in the high temperature regime for all the compounds. The effective moment $\left(\mu_{\mathrm{eff}}=\sqrt{\frac{3 k_{\mathrm{B}} C}{N_{\mathrm{A}}}}\right)$ calculated for $S=5 / 2$ from the value of $C$ and the corresponding value of $g\left[\mu_{\text {eff }}=g \sqrt{S(S+1)}\right]$ are also tabulated

\begin{tabular}{|c|c|c|c|c|c|}
\hline Compound & $\begin{array}{l}\chi_{0} \\
\left(\mathrm{~cm}^{3} \mathrm{~mol}^{-1} \mathrm{Mn}\right)\end{array}$ & $\begin{array}{l}C \\
\left(\mathrm{~cm}^{3} \mathrm{~K} \mathrm{~mol}^{-1} \mathrm{Mn}\right)\end{array}$ & $\theta_{\mathrm{CW}}(\mathrm{K})$ & $\mu_{\mathrm{eff}}\left(\mu_{\mathrm{B}}\right)$ & $g$ \\
\hline 1 & $-2.77 \times 10^{-4}$ & 4.43 & 1.92 & 5.95 & 2.01 \\
\hline 2 & $-3.59 \times 10^{-4}$ & 5.33 & 12.83 & 6.52 & 2.20 \\
\hline 3 & $-7.08 \times 10^{-4}$ & 4.45 & 17.22 & 5.96 & 2.01 \\
\hline 4 & $-3.77 \times 10^{-4}$ & 5.32 & 20.24 & 6.52 & 2.20 \\
\hline 5 & $-2.42 \times 10^{-4}$ & 4.40 & 19.90 & 5.93 & 2.00 \\
\hline 6 & $-2.36 \times 10^{-4}$ & 3.75 & 13.50 & 5.47 & 1.85 \\
\hline 7 & $-4.83 \times 10^{-4}$ & 4.74 & 22.95 & 6.15 & 2.08 \\
\hline 8 & $-1.09 \times 10^{-3}$ & 3.50 & 29.88 & 5.28 & 1.79 \\
\hline
\end{tabular}

increases linearly with $H$ with no sign of saturation even at $H=9 \mathrm{~T}$, as expected. In these two compounds, $\chi(T)$ shows a pronounced broad maximum suggesting that the trimers are interconnected forming 1D antiferromagnetic chains.

In low-dimensional spin systems, the temperature corresponding to the broad maximum $\left(T^{\max }\right)$ reflects the energy scale of the exchange coupling. ${ }^{42}$ Thus, low values of $T^{\max } \simeq 3.16 \mathrm{~K}$, $\theta_{\mathrm{CW}} \simeq 1.92 \mathrm{~K}$ and saturation field $H_{\mathrm{S}} \simeq 4.5 \mathrm{~T}$ suggest that the interaction between the interacting species in compound 1 is very weak. For compounds $\mathbf{1}, \mathbf{2}$ and $\mathbf{6}$, which show clear broad maxima, the $\chi(T)$ data were fitted using

$$
\chi(T)=\chi_{0}+\chi_{\text {spin }}(T)
$$

where $\chi_{\text {spin }}$ is the spin susceptibility of the $S=5 / 2$ onedimensional (1D) chain model. The high-temperature series expansion (HTSE) of $\chi_{\text {spin }}$ for the $S=5 / 2$ Heisenberg 1D chain was reported by Dingle $e t a l .{ }^{43}$

$$
\chi_{\text {spin }}(T)=\frac{N_{\mathrm{A}} g^{2} \mu_{\mathrm{B}}^{2}}{3 k_{\mathrm{B}} T} S(S+1) \frac{1+\mu(K)}{10 \mu(K)}
$$

where

$$
\mu(K)=\operatorname{coth} K-\frac{1}{K}
$$

and $K=-2 J S(S+1) / k_{\mathrm{B}} T$. As shown in Fig. $4 \mathrm{a}, \mathrm{c}$ and $5 \mathrm{c}$, the experimental data down to the broad maxima region were fitted well using eqn (3) and the average exchange coupling $\left(\mathrm{J} / k_{\mathrm{B}}\right)$ between $\mathrm{Mn}^{2+}$ ions along the chains was estimated to be $J / k_{\mathrm{B}} \simeq 0.30 \mathrm{~K}, 0.74 \mathrm{~K}$ and $1.02 \mathrm{~K}$ for compounds 1,2 , and 6 , respectively.

\section{Theoretical analysis}

First principles theoretical studies were carried out to understand the origin of the variations in the optical band gap energy and magnetic properties of compounds 1-8. The optimized structures of the eight solids with their most stable magnetic orientation are shown in Fig. 6. Note that the structures are corrected so that the substituted benzene-1,4-dicarboxylic acid (BDC) based linkers are all singly substituted. To determine the alignment of the spins on different Mn sites, we carried out broken symmetry solutions within the DFT calculations.
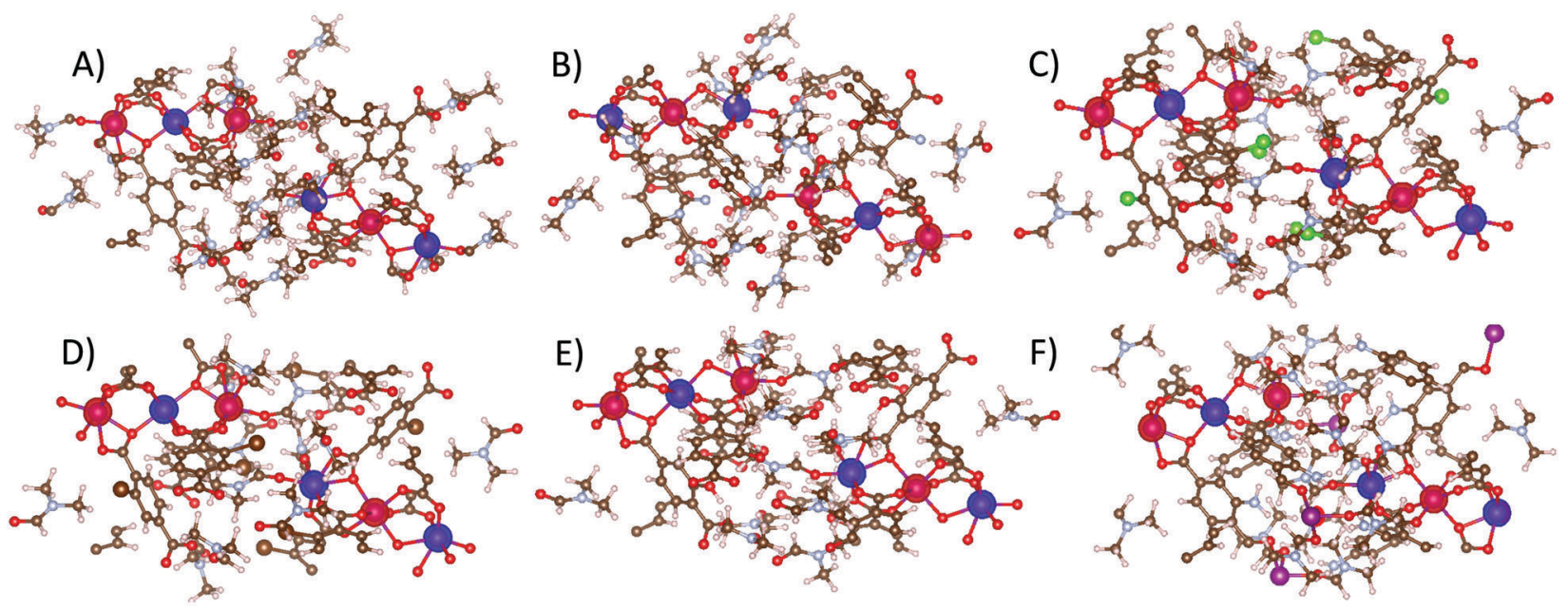

G)
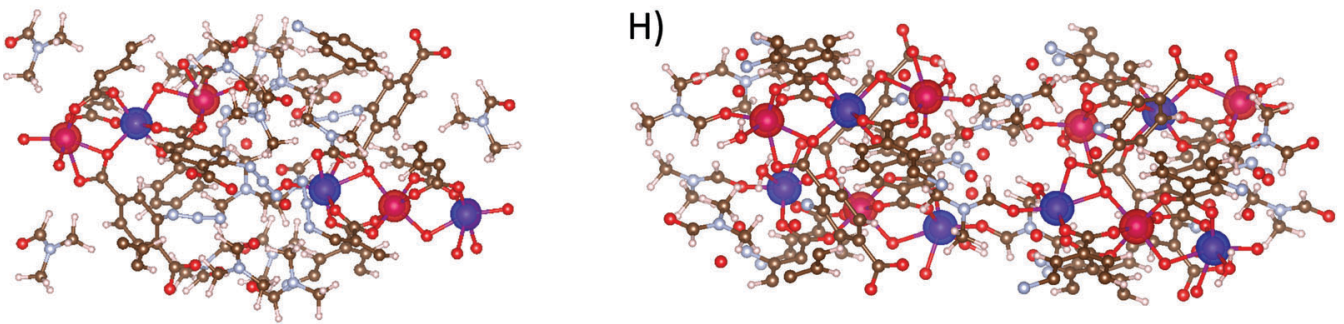

Fig. 6 The structure of the (A) H-based, (B) F substituted, (C) Cl Substituted, (D) Br substituted, (E) OH substituted, (F) $\mathrm{NH}_{2}$ substituted, (G) $\mathrm{N}_{3}$ substituted, and $(\mathrm{H}) \mathrm{NO}_{2}$ substituted linker. The blue isosurface corresponds to alpha spin and the red isosurface corresponds to beta spin. The atoms are colored as follows: $\mathrm{Mn}$ : purple, $\mathrm{C}$ : brown, $\mathrm{N}$ : pale blue, $\mathrm{O}$ : red, $\mathrm{H}$ : white, $\mathrm{F}$ : light blue, $\mathrm{Cl}$ : green, and $\mathrm{Br}$ : brown. 
The investigations included varying the orientation of Mn spins in a single trimer as well as the relative orientation of spins in different trimers. We began by examining the variations in the band gap energies. Here we separated the states localized on the Mn sites and on the ligands through an examination of the projected density of states. This was useful to find out whether the changes in the electronic structure of the Mn trimers or those of the ligands were responsible for the observed variations in the band gap. Fig. 7 shows the projected density of states from compounds 1-8, showing the density projected onto the $\mathrm{Mn}$ atoms, the BDC linker, the dimethylformamide (DMF) solvent molecule, and the substituted functional group. In the $\mathrm{NO}_{2}$ substituted crystal structure, (compound 5), there is an additional set of water molecules bound to the $\mathrm{Mn}$ atom, and these are included in the projected density of states of the solvent. We have plotted the ferromagnetic state to simplify the density of states, and in all cases we observed that the spin down channel has a significant gap, while the spin up channel has a relatively small gap. These values for the alpha and beta band gap energy are given in Table 1. For further comparison, we calculated the HOMO-LUMO gaps for the substituted BDC
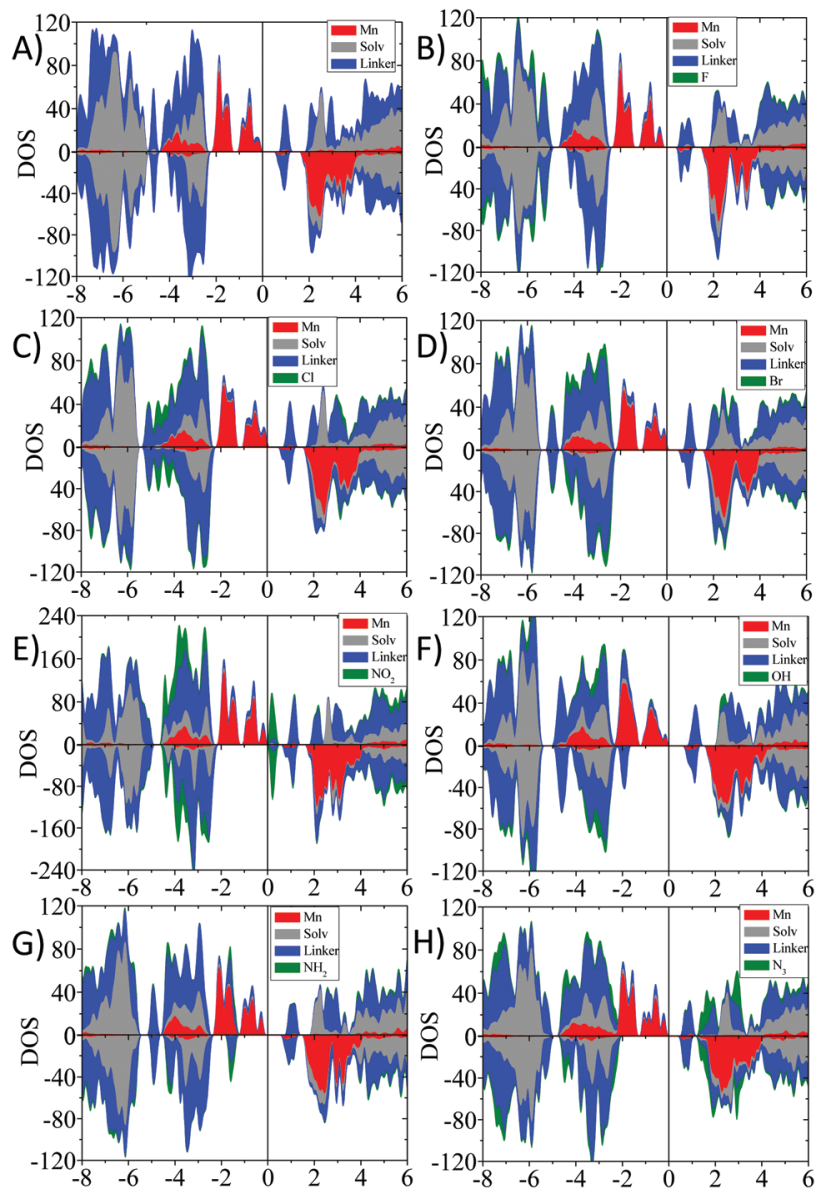

Fig. 7 The projected density of states for the (A) BDC, (B) F substituted, (C) $\mathrm{Cl}$ substituted, (D) $\mathrm{Br}$ substituted, (E) $\mathrm{NO}_{2}$ substituted, (F) $\mathrm{OH}$ substituted, (G) $\mathrm{NH}_{2}$ substituted, and $(\mathrm{H}) \mathrm{N}_{3}$ substituted linker. The ferromagnetic density of states is shown here for clarity. Positive values of DOS are for the alpha spin, and negative values of DOS correspond to beta spin. linkers, also shown in Table 1. First, we observed that the measured band gap energies are generally consistent with the beta spin channel optical band gap energies, while those in the alpha channel are quite low.

The oscillator strength in the alpha channel is also low. This is because the top of the valence band in the alpha spin channel is localized on the Mn sites, while the bottom of the conduction band is localized on the BDC linker. Since the origin of the two electronic states is physically separated, the oscillator strength for these optical excitations is quite weak. To demonstrate this, we calculated the optical absorption spectra for a free cluster (Fig. S4, ESI $\dagger$ ), and we found that there is a small absorption band close to $1.8 \mathrm{eV}$ corresponding to the alpha gap, but the dominant absorption band is at $3.1 \mathrm{eV}$, and corresponds to the electronic excitation between states on the benzene linker. This indicates that the observed transitions are largely due to the beta spin channel, and that the changes in the linkers could account for the variations in the optical band gap energies.

We now examine more critically whether the variations in the measured optical band gap energies can be related to the electronic structure of the substituted BDC linker. As shown in Table 1, the measured band energy corresponds reasonably well to the HOMO-LUMO gap of the BDC molecule. In the series in which BDC is substituted with halogens, we find that the trend from $\mathrm{H}$ to $\mathrm{F}-\mathrm{Br}$ results in a decrease in the band gap energy. By examining the frontier orbitals, we find that F substitution lowers the HOMO by $0.06 \mathrm{eV}$, and the LUMO by $0.14 \mathrm{eV}$, resulting in a small decrease in the HOMO-LUMO gap. $\mathrm{Cl}$ and $\mathrm{Br}$ substitutions result in an increase in the HOMO by $0.12 \mathrm{eV}$ and $0.23 \mathrm{eV}$, respectively. This is most likely driven by the halogens breaking the symmetry of the $\pi$ bonding in the BDC, causing a small decrease in the band gap energy, and the decrease becomes larger as the halogen withdraw more electrons. This is consistent with the small decrease in the band gap energy seen in experiments, with the largest decrease coming from the Br substituted BDC. From the density of states it was observed that the halogens mix with the BDC linker density of states resulting in an increase in the energy of the linker contributed valence band (Fig. 7).

A larger decrease in the band gap energy is observed for the substituted functional groups: $\mathrm{OH}, \mathrm{NH}_{2}, \mathrm{NO}_{2}$, and $\mathrm{N}_{3}$. The hydroxyl substituted BDC results in a decrease in the band gap energy as compared to the pure BDC linker. The activating $\mathrm{OH}$ group pushes up the HOMO of the molecular linker by $0.56 \mathrm{eV}$, which is consistent with a decrease in the measured band gap energy of $0.64 \mathrm{eV}$. This is also seen in Fig. 7, with the valence band, especially in the spin beta channel being pushed up in energy. The $\mathrm{NH}_{2}$ substituted linker increases the position of the HOMO by $1.29 \mathrm{eV}$, while the solid is observed to have a decrease in the band gap energy of $0.84 \mathrm{eV}$. Because the $\mathrm{NH}_{2}$ group is more activating than the $\mathrm{OH}$, the decrease in the band gap energy is larger. The density of states shows that the $-\mathrm{NH}_{2}$ hybridized state has reduced the optical gap even more than $\mathrm{OH}$. In the $\mathrm{N}_{3}$ substituted MOF, the azide inserts a new state in the band gap, resulting in an increase in the HOMO by $0.47 \mathrm{eV}$. 
Furthermore, $\mathrm{N}_{3}$ is also an activating group that lowers the position of the conduction band, resulting in an even lower experimental band gap energy of $2.62 \mathrm{eV}$. In the density of states, this state mixes with the linker states resulting in a calculated optical band gap energy of $2.70 \mathrm{eV}$. In the $\mathrm{NO}_{2}$ substituted BDC, the nitro group inserts a new state into the gap, which causes a significant decrease in the band gap energy. The bottom of the valence band is now localized on the $\mathrm{NO}_{2}$ group and the presence of $\mathrm{NO}_{2}$ lowers the conduction band by $0.65 \mathrm{eV}$. This is consistent with the experimental results where the band gap energy is decreased by $0.40 \mathrm{eV}$. Thus, the optical band gap energy of the substituted BDC- $\mathrm{Mn}_{3}$ MOFs may be understood by analyzing the frontier orbitals of the substituted BDC, and the largest shifts occur when the substituted functional group introduces a new state inside of the band gap. Furthermore, by selecting the substituted group, the band gap energy of the MOFs may be systematically tuned from $2.62 \mathrm{eV}$ to $3.57 \mathrm{eV}$, while maintaining the same $\mathrm{Mn}_{3}$ cluster motif.

To examine the magnetic properties, we first considered the orientation of spins on different Mn sites. In all cases, the most stable spin configuration corresponds to antiferromagnetic coupling on the Mn sites within each trimer. In the unit cell of all of the materials except for the $\mathrm{NO}_{2}$ substituted $\mathrm{BDC}$, the unit cell has two $\mathrm{Mn}_{3}$ clusters, and in all cases the most stable configuration is for each $\mathrm{Mn}_{3}$ trimer to exhibit antiferromagnetic coupling, with the central atom having the spin aligned opposite to the two outside atoms. Because there are two $\mathrm{Mn}_{3}$ clusters in each unit cell, the most stable configuration is when the net moment on each $\mathrm{Mn}_{3}$ cluster is antiferromagnetically coupled to each other leading to a net spin moment of $0 \mu_{\mathrm{B}}$. The material with $\mathrm{NO}_{2}$ as the substituent has four $\mathrm{Mn}_{3}$ clusters in the unit cell, and here too each cluster is antiferromagnetic within itself and the other clusters, and the $0 \mu_{\mathrm{B}}$ total spin is the most stable. The typical net spin on each $\mathrm{Mn}$ atom calculated using a Mulliken population is about $4.45 \mu_{\mathrm{B}}$, and spin configurations that did not correspond to a multiple of $5 \mu_{\mathrm{B}}$ for the Mn atom resulted in a significant decrease in stability. The net charge on a typical $\mathrm{Mn}$ atom is calculated to be $+1.55 e^{-}$, and both of these results are consistent with $\mathrm{Mn}$ being in the +2 oxidation state.

To understand the magnetic properties we investigated the relative energy of states with different magnetic couplings. Table 3 shows the energy in meV as a function of the alignment of the six Mn atoms in the unit cell. As mentioned earlier, for individual $\mathrm{Mn}_{3}$ units, the antiferromagnetic arrangement is the most stable, with the central atom aligned opposite to the outside atoms. The interaction between the different $\mathrm{Mn}_{3}$ units is however weak.

While the ground state has the net spins aligned opposite to each other, it takes only $2.9-5.8 \mathrm{meV}$ to have a state where the net spins are aligned with each other leading to a spin moment of $10 \mu_{\mathrm{B}}$. To compare this with the strength of the interaction between Mn sites within a trimer, we considered a state of $10 \mu_{\mathrm{B}}$ but where the two spins within a trimer were aligned parallel to each other. These states are around 13-21 meV higher in energy. A ferromagnetic state corresponding to completely
Table 3 The energy in meV for the different spin states of the MOFs. The unit cell of the MOFs contain $6 \mathrm{Mn}$ atoms, so the spin state corresponds to the alignment of the spin in the first and second $\mathrm{Mn}_{3}$ clusters

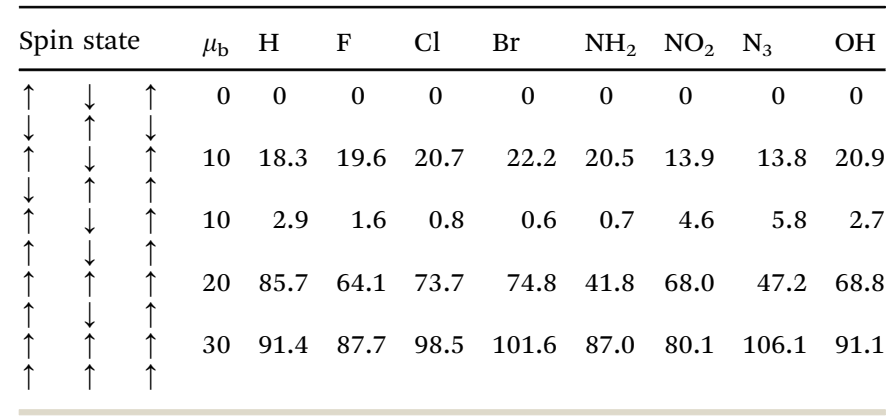

aligned spin moments is only 80-102 meV higher in energy, which corresponds to an average energy of $15 \mathrm{meV}$ per atom. In all cases, there is no magnetization at low temperatures, which is also consistent with the antiferromagnetic arrangement of moments on separate $\mathrm{Mn}_{3}$ clusters. We now consider the observation of plateaus at a magnetization of $\approx 1.67 \mu_{\mathrm{B}}$, which is much below the saturation magnetization of $5 \mu_{\mathrm{B}}$. We believe that this could be a signature of weaker inter-cluster coupling compared to intra-cluster coupling. As mentioned above, theoretical studies indicate that for a pair of $\mathrm{Mn}_{3}$ clusters, the state with a total moment of $10 \mu_{\mathrm{B}}$ is only $4 \mathrm{meV}$ or $0.67 \mathrm{meV}$ per atom higher in energy than the anti-ferromagnetic state. It is then reasonable that as the field is applied, the clusters are first excited to this state which corresponds to a moment of $1.67 \mu_{\mathrm{B}}$ per atom, which is close to the observed value. Compound 1 has a larger moment per atom of $5 \mu_{\mathrm{B}}$, showing that the coupling within the cluster may be weaker for compound $\mathbf{1}$ than for the other compounds. The theoretical results do not agree with this result. One possibility is that the voids in compound $\mathbf{1}$ are larger than those in the other compounds, so $\mathrm{Mn}$ atom impurities may affect the results, although we do not see evidence for paramagnetic impurities in the magnetic susceptibility data as we do in compound 2.

While the spins on each $\mathrm{Mn}$ in the $\mathrm{Mn}_{3}$-based units show antiferromagnetic coupling, we wanted to examine whether the spins are directed along the line joining the three $\mathrm{Mn}$ atoms. We investigated the energy of the system as a function of $\theta$ and $\phi$, as shown in Fig. S5 (ESI $\dagger$ ). The lowest energy (easy axis) corresponds to the spins pointing almost perpendicular to the line joining the atoms (there are two minima corresponding to $\theta=75^{\circ}$ by $\phi=150^{\circ}$ and $\theta=105^{\circ}$ by $\phi=330^{\circ}$. However, the surface is quite shallow and the change in energy from 90 degrees is fairly small). The anisotropy energy of the system is only $0.8 \mathrm{~K}$, which is smaller than the intra-atomic exchange energy. It is then likely that the clusters first undergo super-paramagnetic relaxations until there is enough energy to break the intra-cluster interactions.

\section{Conclusions}

We have systematically synthesized a series of eight MOFs with Mn trimer as SBU and differently substituted BDC as the linker. 
We observed variations in the optical band gap energy, and theoretical studies indicate that these variations are caused by changes in the linker. These changes in the linker add new states to the conduction band that lower the band gap energy. This demonstrates that by varying the functional group bound to the BDC, the optical band gap energy may be varied even in a MOF that contains a metal cluster with d electrons. The assemblies also display interesting magnetic behaviors, including weak anti-ferromagnetism at low temperatures and a paramagnetic behavior above $10 \mathrm{~K}$. Experimental and theoretical studies show that the MOFs are antiferromagnetic, with weak coupling between the trimers that allows the saturation moment to be approximately $1.67 \mu_{\mathrm{B}}$. The compounds exhibit paramagnetic behaviors above $10 \mathrm{~K}$, with five of the compounds being paramagnetic down to $2.1 \mathrm{~K}$. Finally, the Mn spin moments are perpendicular to the line joining the Mn sites and the system is marked by a magnetic anisotropy energy of $0.8 \mathrm{~K}$, which could lead to superparamagnetic relaxations eventually transforming to a conventional paramagnet.

\section{Funding sources}

Science and Engineering Research Board (SERB), Govt. of India, through a grant SB/S1/IC-14/2013.

\section{Acknowledgements}

SM acknowledges the Science and Engineering Research Board (SERB), Govt. of India, for funding through the grant SB/S1/IC14/2013. ACR and SNK gratefully acknowledge support from the US Department of Energy (DOE) through grant DE-SC 0006420 for this work. We are grateful to Prof. V. Ramakrishnan for the encouragement and support. AKS acknowledges CSIR for the fellowship.

\section{References}

1 J. R. Long and O. M. Yaghi, Chem. Soc. Rev., 2009, 38, 1213.

2 A. U. Czaja, N. Trukhan and U. Müller, Chem. Soc. Rev., 2009, 38, 1284.

3 U. Schubert, Chem. Soc. Rev., 2011, 40, 575.

4 A. Corma, H. García and F. X. Llabrés i Xamena, Chem. Rev., 2010, 110, 4606.

5 R. L. Martin and M. Haranczyk, Chem. Sci., 2013, 4, 1781.

6 E. Flage-Larsen and K. Thorshaug, Inorg. Chem., 2014, 53, 2569.

7 M. E. Foster, J. D. Azoulay, B. M. Wong and M. D. Allendorf, Chem. Sci., 2014, 5, 2081.

8 M. D. Allendorf, A. Schwartzberg, V. Stavila and A. A. Talin, Chem. - Eur. J., 2011, 17, 11372.

9 S. B. Darling and S. D. Bader, J. Mater. Chem., 2005, 15, 4189.
10 M. Kurmoo, Chem. Soc. Rev., 2009, 38, 1353.

11 K. S. Asha, K. M. Ranjith, A. Yogi, R. Nath and S. Mandal, Dalton Trans., 2015, 44, 19812.

12 M. C. Bernini, J. R. de Paz, N. Snejko, R. Sáez-Puche, E. Gutierrez-Puebla and M. Á. Monge, Inorg. Chem., 2014, 53, 12885.

13 C. K. Brozek and M. Dincă, J. Am. Chem. Soc., 2013, 135, 12886.

14 Q. Zhang, B. Li and L. Chen, Inorg. Chem., 2013, 52, 9356.

15 M. Zhang, H. Zhang, L. Zhao, Y. Li and Y. Luo, J. Phys. Chem. A, 2012, 116, 1493.

16 L. Li, G. J. Clarkson, D. J. Evans, M. R. Lees, S. S. Turner and P. Scott, Chem. Commun., 2011, 47, 12646.

17 X.-L. Tong, T.-L. Hu, J.-P. Zhao, Y.-K. Wang, H. Zhang and X.-H. Bu, Chem. Commun., 2010, 46, 8543.

18 D. W. Ryu, W. R. Lee, J. W. Lee, J. H. Yoon, H. C. Kim, E. K. Koh and C. S. Hong, Chem. Commun., 2010, 46, 8779.

19 E. D. Bloch, W. L. Queen, R. Krishna, J. M. Zadrozny, C. M. Brown and J. R. Long, Science, 2012, 335, 1606.

20 S. Mohapatra, B. Rajeswaran, A. Chakraborty, A. Sundaresan and T. K. Maji, Chem. Mater., 2013, 25, 1673.

21 P. Mahata, D. Sarma, C. Madhu, A. Sundaresen and S. Natarajan, Dalton Trans., 2011, 40, 1952.

22 D. Sarma, P. Mahata, S. Natarajan, P. Panissod, G. Rogez and M. Drillon, Inorg. Chem., 2012, 51, 4495.

23 P. Mahata, S. Natarajan, P. Panissod and M. Drillon, J. Am. Chem. Soc., 2009, 131, 10140.

24 D. Maspoch, D. Ruiz-Molina and J. Veciana, J. Mater. Chem., 2004, 14, 2713.

25 R. Dingle, M. E. Lines and S. L. Holt, Phys. Rev., 1969, 187, 643.

26 S.-Q. Bai, E.-Q. Gao, Z. He, C.-J. Fang, Y.-F. Yue and C.-H. Yan, Eur. J. Inorg. Chem., 2006, 407.

27 H. J. Eppley, H.-L. Tsai, N. de Vries, K. Folting, G. Christou and D. N. Hendrickson, J. Am. Chem. Soc., 1995, 117, 301.

28 J. R. Friedman, M. P. Sarachik, J. Tejada and R. Ziolo, Phys. Rev. Lett., 1996, 76, 3830.

29 M. R. Pederson and S. N. Khanna, Phys. Rev. B: Condens. Matter Mater. Phys., 1999, 60, 9566.

30 M. R. Pederson and S. N. Khanna, Phys. Rev. B: Condens. Matter Mater. Phys., 1999, 59, R693.

31 M. Alvaro, E. Carbonell, B. Ferrer, F. X. Llabrés i Xamena and H. Garcia, Chem. - Eur. J., 2007, 13, 5106.

32 S. Mandal, A. C. Reber, M. Qian, P. S. Weiss, S. N. Khanna and A. Sen, Acc. Chem. Res., 2013, 46, 2385.

33 J. H. Choi, H. J. Jeon, K. M. Choi and J. K. Kang, J. Mater. Chem., 2012, 22, 10144.

34 K. S. Asha, A. C. Reber, A. F. Pedicini, S. N. Khanna and S. Mandal, Eur. J. Inorg. Chem., 2015, 2085.

35 R. Ghosh, A. F. Pedicini, P. C. Rao, K. S. Asha, A. C. Reber and S. Mandal, Dalton Trans., 2015, 44, 13464.

36 J.-L. Wang, C. Wang and W. Lin, ACS Catal., 2012, 2, 2630. 
37 M. Qian, A. C. Reber, A. Ugrinov, N. K. Chaki, S. Mandal, H. M. Saavedra, S. N. Khanna, A. Sen and P. S. Weiss, ACS Nano, 2010, 4, 235.

38 S. Mandal, A. C. Reber, M. Qian, R. Liu, H. M. Saavedra, S. Sen, P. S. Weiss, S. N. Khanna and A. Sen, Dalton Trans., 2012, 41, 12365.

39 C. Kittel, Introduction to solid state physics, Wiley India, 2005 , ch. 12 , p. 325.
40 P. W. Selwood, Magnetochemistry, Interscience Publishers Newyork, 1942, ch. 4, p. 98.

41 Y. Lee, et al., Phys. Rev. Lett., 2015, 115, 167203.

42 K. H. J. Buschow, Handbook of magnetic materials, 1997, ch. 1, vol. 10, p. 34 .

43 A. C. Reber, S. Mandal, M. Qian, H. M. Saavedra, P. S. Weiss, S. N. Khanna and A. Sen, J. Phys. Chem. C, 2012, 116, 10207. 